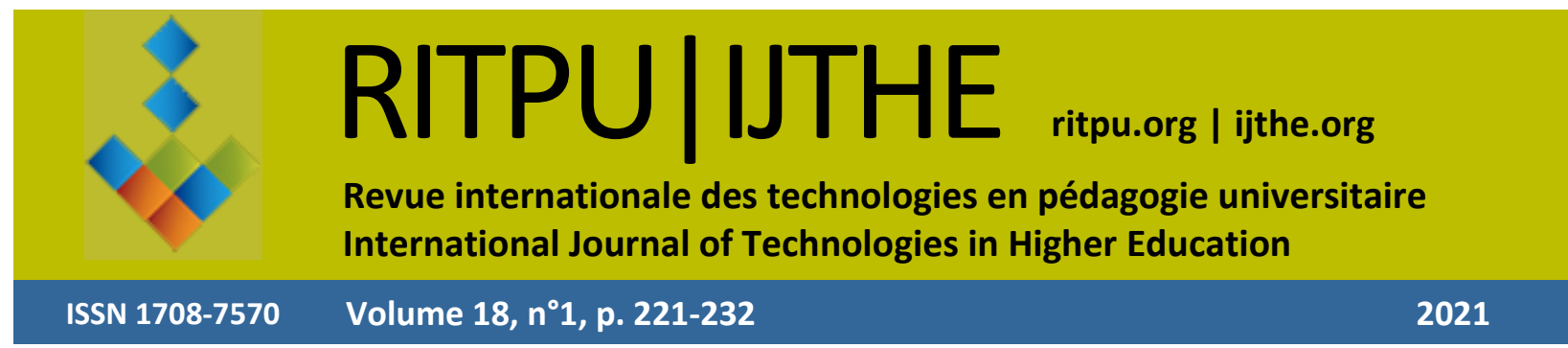

\title{
Leçons et questions de la continuité pédagogique dans une formation française d'ingénieurs
}

\author{
Nadine DUBRUC \\ dubruc@emse.fr \\ Mines Saint-Étienne ${ }^{1}$ \\ France
}

\author{
Lessons Learned and Pedagogical Continuity in an \\ Engineering Training Program in France
}

https://doi.org/10.18162/ritpu-2021-v18n1-19

\section{Mis en ligne : 9 avril 2021}

\section{Résumé}

En France, à cause d'un virus, mars 2020 marquera pour beaucoup d'enseignants le début d'une expérience d'enseignement à distance à l'aide des outils numériques. Assurer la continuité pédagogique s'impose comme une évidence. Zoom devient la nouvelle salle de classe. Un retour d'expérience dans le cadre d'une formation d'ingénieurs permet de tirer quelques enseignements de cette période. Les leviers de cette continuité sont l'adaptation de l'animation en fonction des objectifs pédagogiques; la formation des enseignants aux outils numériques accessibles; des actions pour fédérer une communauté dispersée dans l'espace; l'adaptation de la règle de la participation en classe (se montrer ou rester caché); la division très visible du temps d'apprentissage; et le développement des interactions dans la communauté. Cette expérience met également en évidence l'impact du numérique sur la perception du temps, la gestion de la fatigue générée par les outils numériques et l'organisation de temps synchrones et asynchrones.

\section{Mots-clés}

Continuité pédagogique, école d'ingénieurs française, instruments, règles, communauté

\section{Abstract}

In France, because of a virus, March 2020 will remain for many teachers the beginning of a distance learning experience using digital tools. Ensuring pedagogical continuity is a matter of course. Zoom becomes the new classroom. Feedback in the context of an engineering training course allows us to draw some lessons from this period. The levers of this continuity are the training of teachers in the digital tools; the adaptation of the animation according to the

1. Affiliation complète : Mines Saint-Etienne, Université de Lyon, Université Lumière, Université Jean Monnet, EA 4161 COACTIS, Institut Henri Fayol. 
pedagogical objectives; actions to federate a community dispersed in space; the adaptation of the rule of participation in class (show yourself or remain hidden); the highly visible division of learning time; and the development of interactions in the community. This experiment also highlights the impact of digital technology on perception of time, management of tiredness generated by digital tools, and the organization of synchronous and asynchronous time.

\section{Keywords}

Pedagogical continuity, French engineering school, instruments, rules, community

\section{Introduction}

En France, mars 2020 marquera pour beaucoup d'enseignants le début d'une expérience d'enseignement à distance à l'aide des outils numériques. En quelques jours, l'année scolaire est bouleversée par un virus, le SRAS-CoV-2. Assurer la continuité pédagogique s'impose comme une évidence. Zoom devient la nouvelle salle de classe. En 2018, Karsenti affirmait «Dans un monde où la place du numérique s'affirme chaque jour davantage, et où les jeunes - et moins jeunes - sont captivés par les technologies, la pédagogie universitaire ne semble avoir d'autre choix que de voir ses méthodes ou pratiques évoluer» (p. 5). Cependant, cela ne s'est pas fait tout seul et ne résultait pas d'un choix. Il aura fallu cette période imposée de confinement et la fermeture des établissements aux étudiants avec l'engagement d'une continuité pédagogique pour faire entrer le numérique dans les pratiques pédagogiques. L'évolution des pratiques pédagogiques a été imposée plus par un contexte externe à l'enseignement que pour répondre au besoin d'un public. Cet élément a-t-il eu une incidence sur l'organisation d'un enseignement?

En tant qu'enseignante mobilisée par des enseignements dans une formation d'ingénieurs généralistes française sur cette période, nous proposons un retour d'expérience distanciée pour tirer les leçons et pointer les questions de cette continuité pédagogique basée sur le numérique. Dans une première partie, nous présentons le contexte de cette expérience pour, dans une deuxième partie, nous interroger sur la facilité de ce passage et proposer un cadre théorique de l'analyse. La troisième partie propose quelques leçons sur l'usage du numérique en pédagogique pour finir par une partie portant sur l'impact de l'enseignement à distance sur la notion de temps.

\section{Contexte du retour d'expérience}

Ce retour d'expérience est réalisé à partir d'une formation d'ingénieurs généralistes de l'École nationale supérieure des Mines de Saint-Étienne (Mines Saint-Étienne) et plus particulièrement de trois modules de cours d'un de ses cycles. Membre de l'Institut Mines-Télécom et rattachée au Ministère chargé de l'industrie, l'École des mines de Saint-Étienne est une des écoles prestigieuses d'ingénieurs de France. Créée en 1816, elle offre plusieurs cycles de formation proposant six cursus dont cinq sont orientés vers des spécialités de haut niveau et un historique axé sur la formation d'ingénieurs généralistes. Ce cycle Ingénieur civil des Mines (ICM) se déroule sur le site de Saint-Étienne, en région Auvergne Rhône-Alpes en France. En 2019, 590 élèves sont inscrits dans ce cursus, répartis sur trois années d'études qui correspondent aux années $b a c+3$ à $b a c+5$. «Avec la formation ICM, l'École réaffirme son ambition de former de futurs leaders et dirigeants avec un profil international au service du développement économique et industriel de notre pays » (Mines Saint-Étienne, s.d., section Développez les 7 qualités de l'ICM pour vous démarquer dans le monde professionnel). Alors que $80 \%$ des étudiants arrivent dans ce cursus par concours après un passage de deux ans en classes préparatoires aux grandes 
écoles, $20 \%$ sont des admis sur titres, pour la majorité provenant d'établissements étrangers partenaires et pour une minorité, d'autres cursus comme pharmacien ou médecin qui ont pour objectif un double diplôme (MESRI, 2020).

Le 13 mars 2020, nous sommes informés que «[d]ans le cadre de la gestion de l'épidémie de COVID-19, l'école sera fermée aux élèves à partir de lundi » (responsable du cycle, communication personnelle). Un module d'ouverture à destination des premières années (environ 150 étudiants) a été lancé ce jour-là par une conférence en présentiel. Une première partie devait se dérouler du lundi 16 mars au mercredi 18 mars puis du mercredi 15 avril au vendredi 17 avril. La première partie est purement et simplement annulée. La deuxième partie sera assurée à distance.

Le 16 mars 2020, le directeur du cycle nous envoie des premières recommandations pour la mise en œuvre du plan de continuité pédagogique. Nous devons mettre sur une plateforme pédagogique existante tous les supports de cours des enseignements qui devraient se dérouler afin de les rendre accessibles aux étudiants. En tant qu'enseignants et responsables d'enseignements, nous sommes mobilisés à double titre : assurer l'accès des supports pour les cours et assurer l'accès aux cours des enseignements supervisés. L'urgence génère des demandes qui vont évoluer au fur et à mesure de la mise en place du plan de continuité.

Dès le 16 mars, la plateforme Zoom est testée et désignée dès le lendemain comme " outil retenu pour l'animation à distance des cours ». "L'idée générale est, pour les cours “classiques" (cours, travaux dirigés), de prévoir un créneau d'animation à distance pour chaque créneau initialement prévu dans le planning initial » (responsable du cycle, communication personnelle, 17 mars 2020). Dès lors, le pôle pédagogique (trois personnes) propose une assistance à la scénarisation pédagogique et à la mise en place technique autour des outils numériques. Le 19 mars, un plan de continuité pédagogique est établi par la direction des formations. Dès le lundi 23 mars, les étudiants reçoivent un courriel indiquant : "La direction de la formation est totalement mobilisée, avec les équipes enseignantes et les services support, pour permettre une poursuite de votre formation dans les meilleures conditions possible en ces circonstances exceptionnelles. » Ainsi est lancée la formation à distance du cycle « Ingénieur civil des Mines de Saint-Étienne ».

\section{Un passage facile au numérique?}

Fourgous (2010) détermine trois leviers principaux pour «réussir l'école numérique »: les élèves (issus de la génération des «enfants du numérique »), les enseignants, les ressources mises à la disposition des enseignants. Nous pourrions désormais ajouter un quatrième levier qui a été le levier principal de ce basculement en 2020: une crise sanitaire qui oblige tous les enseignants à adopter le numérique sur une période de trois à six mois. Effectivement, nous voyons bien que ce qui a été décisif dans ce basculement a été la contrainte du confinement sanitaire et la volonté de poursuivre l'activité d'enseignement sur une durée indéterminée. Dans ce cycle de formation, la décision a été prise très rapidement d'assurer une continuité pédagogique en utilisant une ressource existante peu utilisée par les enseignants : Moodle, une plateforme pédagogique pour a minima partager des documents. Ce dispositif est complété par une nouvelle ressource : une plateforme qui permet de réaliser des cours en visioconférence : Zoom. Lollia et Issaieva (2020) indiquent qu'une variété d'outils numériques a été mobilisée par les enseignants de manière complémentaire : site Internet, applications WhatsApp, Skype... Tandis que le ministère de l'Éducation nationale français déconseillait l'utilisation de Zoom, notre établissement dépendant du ministère de l'Industrie a fait le choix de cet outil. Celui-ci est 
largement utilisé en interne car les cours ont été programmés sur cette plateforme par les services administratifs. C'est donc pour cela que nous avons choisi de nous focaliser sur l'adaptation des enseignements à cet outil en laissant de côté les autres options. Dans cette période étrange, il n'y a pas eu possibilité de discuter des outils choisis. Même si ces derniers ont été imposés, chaque enseignant a pu suivre les directives tout en ayant la possibilité d'utiliser autre chose. Dans notre établissement, l'été 2020 a permis au service informatique et à la direction de la formation de faire de nouvelles propositions d'outils à partir du critère de garantie de sécurité.

Il est quand même étonnant de constater comment, dans ce contexte, il semble y avoir eu un ordre de marche, non contesté par les enseignants ni par les directions des établissements d'enseignement supérieur. Partir de l'idée qu'en une ou deux semaines, une formation complètement en présentiel puisse se transformer en formation à distance ne serait certainement pas passé aussi simplement en temps normal. Dans cette situation d'urgence, exceptionnelle, la logique qui a prévalu a été celle de faire «comme si » : comme si tout pouvait continuer comme avant le confinement, comme si on pouvait passer d'une modalité pédagogique à une autre naturellement, comme si les étudiants pouvaient s'adapter à une autre façon d'apprendre, à distance tranquillement... comme si on avait tous oublié quelques principes d'apprentissage et de pédagogie. Et dans ce contexte, un élément de langage a été tout particulièrement irritant: « enseignement en mode dégradé », une expression difficile à entendre pour un enseignant qui prend un temps important pour reconcevoir son ingénierie pédagogique et qui est satisfait du travail fourni, avec notamment de bons retours des étudiants, contents de ces cours reconçus en mettant en avant l'interactivité permise par la visioconférence... Utilisée régulièrement par la direction de la formation pour excuser une situation inédite, l'expression « enseignement en mode dégradé » a selon nous nié et n'a pas reconnu le grand travail d'adaptation réalisé par certains enseignants.

Pour les enseignants, faire leur travail, poursuivre les cours commencés et ne pas mettre en jeu une partie de l'année scolaire d'une génération ont été autant de motivations. Assurer la continuité pédagogique n'a pas été contesté dans notre établissement. L'accompagnement à la transformation des cours a permis à certains d'être plus proactifs et de se former pour opérer des changements dans leurs pratiques pédagogiques. Seule une minorité n'a pas suivi le mouvement et s'est contentée de déposer les supports de cours sur la plateforme mise à la disposition des étudiants sans plus de suivi. Le bilan réalisé à l'issue d'une période de cours avec la finalisation de certains contenus pédagogiques fait ressortir que ce dernier cas de figure n'a pas forcément été apprécié des étudiants. Par exemple, dans un module, un cours de droit social a été délivré avec le dépôt de documents sur la plateforme et des échanges de courriels entre les étudiants et le responsable du module. L'intervenant en droit n'avait pas l'équipement nécessaire pour réaliser des visioconférences. Les séances ont été organisées à partir de documents (arrêtés de justice) dont les étudiants devaient faire des synthèses sur un thème : le contrat de travail, le contrat de travail à l'international. Ce cours a commencé au début du confinement et le responsable et l'intervenant espéraient toujours que le prochain cours se fasse en présentiel... au moins le premier mois... Finalement, les retours des étudiants sur ce cours ont été très négatifs alors que c'est un cours qui faisait l'unanimité les années précédentes en présentiel... En résumé, voici une remarque d'une étudiante : «Le cours sur le droit social était très moyen. J'ai l'impression de [ne] rien avoir appris. Le fait de juste donner des décisions d'assise n'est pas très pédagogique je trouve. J'en ressors juste avec la connaissance de comment une décision d'assise est structurée. » N'avons-nous pas oublié d'aligner les objectifs pédagogiques sur les modalités pédagogiques?

De même, dans ce module, il est prévu un enseignement qui fait le parallèle entre le management et le jeu de go. Le cours est construit en présentiel à partir de séances au jeu de go... 
L'intervenante a revu son animation pour pouvoir la réaliser sur Zoom avec une alternance de séquences en grand groupe et d'autres en minigroupes, mais n'a pas pu maintenir des parties de jeu. Dans le bilan, cette exclamation est ressortie : « À cause du confinement, nous aurions bien aimé jouer au go en vrai! » Il en ressort un sentiment d'éléments donnés qui sonneraient faux... ou tout du moins qui ont peut-être eu du mal à entrer dans la réalité de cet étudiant. Et cela renvoie aux raisons qui font choisir ce type d'activité pédagogique en présentiel : faire faire aux étudiants afin que l'activité proposée s'ancre dans un apprentissage par la pratique. Faire et comprendre à partir de cette expérience et apprendre d'elle n'a pas pu être réalisé dans cette situation. À la suite de ce bilan et aux échanges avec les étudiants qui ont unanimement partagé ce retour, une solution éventuelle a été trouvée pour pouvoir faire jouer des parties à distance... Ces deux cas représentent deux exemples de différentes façons d'appréhender cette continuité pédagogique numérique imposée par la COVID-19.

Quelles leçons pouvons-nous tirer de cette expérience Zoom/COVID-19? Quels sont les éléments essentiels qui s'imposent lors du passage au tout numérique dans une formation d'ingénieurs faite habituellement en présentiel? Et comment certains leviers d'organisation d'une activité pédagogique se heurtent-ils à des questions de temps et de lieux pour les étudiants et les enseignants?

\section{Leçons de la continuité pédagogique}

Nous avons voulu approfondir notre réflexion en analysant notre expérience de cette transformation numérique à l'aide du cadre théorique de l'apprentissage vue comme un système d'activité selon Engeström (1987, 2006) et Vygotski (1934/2002). Ainsi, nous considérons que toute activité se réalise à l'aide d'instruments (physiques ou psychologiques) et à partir de règles, explicites ou implicites, définies par la communauté selon une division du travail qui définit la répartition des tâches pour réaliser les actions selon différentes responsabilités. Un enseignement serait une activité réalisée par un enseignant pour un sujet ou des sujets faisant partie d'une communauté et ayant pour objet la transmission d'un thème et pour objectif l'acquisition de connaissances ou de compétences en fonction de règles et selon une division du travail en appui sur des instruments mobilisés. Ainsi, un enseignant mobilise un instrument qui serait Zoom selon certaines règles et une division du travail dans une communauté pour favoriser un apprentissage. Nous reprenons la représentation de l'activité d'enseignement suivante (figure 1) :

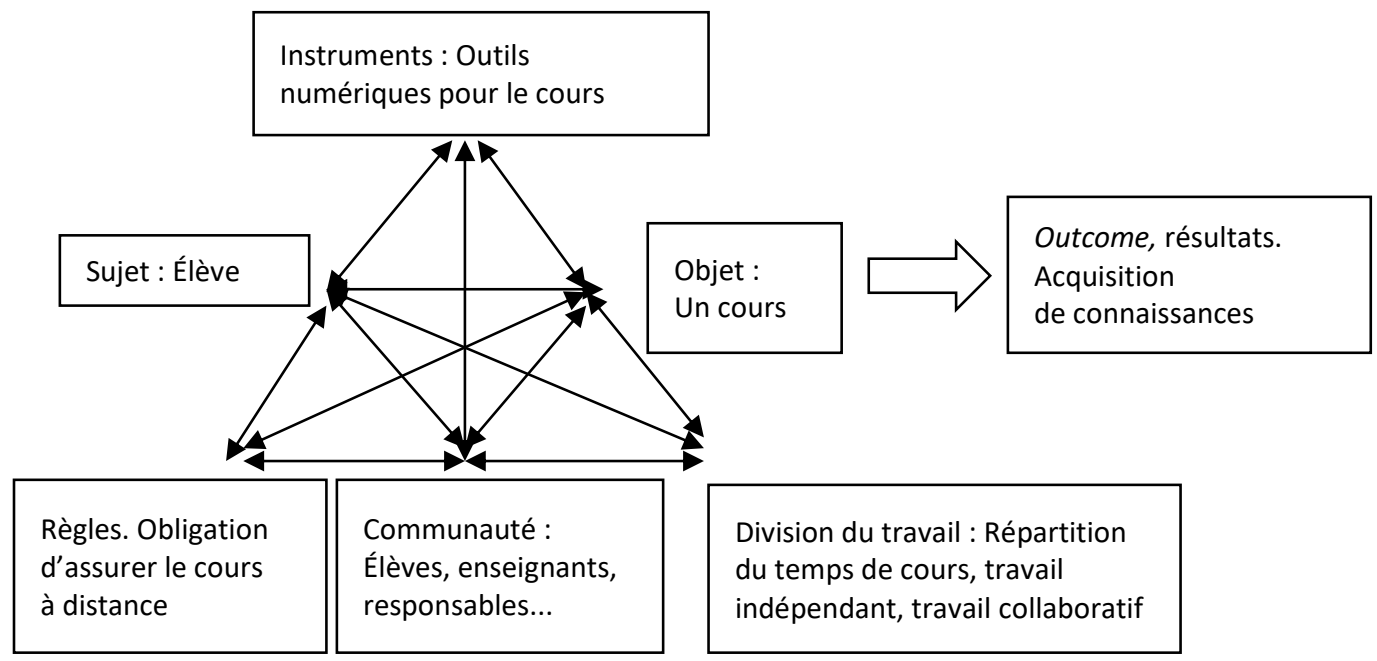

Figure 1

Structure de l'activité humaine (d'après Engeström, 1987) 
Cette structure nous a permis d'organiser notre analyse réflexive sur cette période vécue et d'en tirer des leçons organisées selon ces éléments. Avec une vision de l'apprentissage dans une approche socioconstructiviste, depuis plus de 20 ans d'enseignement, nos pratiques sont basées sur la pédagogie active. Ainsi, «chaque apprenant dispose de connaissances (des conceptions) et de compétences avec lesquelles il va construire des connaissances nouvelles pour résoudre des problèmes que lui pose l'environnement. Le rôle de la communauté — les autres étudiants et l'enseignant - est de fournir le dispositif, de mettre en lumière le défi et de le soutenir dans la construction personnelle qu'il entreprend » (Lebrun, 2007, p. 35). Nos enseignements reposent sur une pédagogie orientée sur le faire faire aux apprenants avec un temps de construction des savoirs à partir de cette expérience, de conceptualisation ce qu'ils ont fait. Pour nous, la question principale a été : Comment passer d'un cours dans la salle 221 à Saint-Étienne à la salle Zoom 13, soit réaliser son enseignement prévu pour un lieu physique dans une salle virtuelle sur Zoom?

Nous proposons d'observer les changements réalisés dans un de nos enseignements et de les parcourir à l'aide de la structure de l'activité. Il s'agit d'un cours de 15 heures en management durable dans un module de gestion des ressources humaines. Nos séances sont basées sur des animations pédagogiques variées qui vont d'un jeu sérieux sur l'entretien annuel à du temps d'animation et de réflexion sur l'analyse de problèmes ou à des restitutions individuelles d'un questionnaire de personnalité. Par exemple, en présentiel, le cours sur la qualité de vie au travail en entreprise se fait à partir d'un jeu de cartes qui permet d'expérimenter une grille d'analyse des situations de travail. Ainsi, ce cours a été reconçu à partir de vidéos sur un cas d'entreprise. Nous avons gardé le travail en sous-groupes pour permettre des échanges, un croisement d'analyses et des compléments d'analyse apportés par les pairs. Puis en grand groupe, nous avons repris les éléments compris et apporté des éclairages complémentaires. Mais finalement, quelles sont les leçons que nous tirons de cette expérience et de toutes les adaptations réalisées pour permettre la réalisation du cours de 15 heures? Pour notre analyse, nous avons repris les courriels échangés entre collègues, où nous faisons part de nos questions et réflexions, et les verbatim des étudiants écrits dans les fiches d'évaluation de l'enseignement. Le cours est enseigné à 20 étudiants de $1^{\text {re }}$ et $2^{\mathrm{e}}$ année d'école d'ingénieurs et s'est déroulé de mars 2020 à mai 2020. Ce retour d'expérience personnel a été confronté en juillet lors d'une réunion d'échange d'expériences avec les autres enseignants-chercheurs de notre département. Sur 10 enseignants-chercheurs présents, nous étions 6 à avoir eu à réaliser des enseignements transformés. Les 4 autres enseignants n'avaient pas de cours ce semestre. Nous étions tous motivés par des échanges pour comprendre ce qui s'était passé dans ce temps atypique d'adaptation imposée. Nous avons procédé à une analyse des éléments qui avaient fonctionné et de nos constats.

Nous allons présenter les leçons tirées chronologiquement selon leur temps d'apparition et la façon dont nous avons progressivement appris à transformer notre enseignement.

- Leçon 1 : se former aux outils numériques accessibles. La première chose accomplie au début du confinement a été une formation sur les outils comme Zoom pour en comprendre les possibilités et les limites. Des webinaires et des réunions de travail entre collègues ont permis cela. Avant la maîtrise qui est venue avec la pratique... nous avons cherché à connaître l'outil qui allait devenir l'instrument principal de nos pratiques pédagogiques numériques. Ainsi, la subtilité de ce qui peut être proposé vient avec la pratique... Se sentir à l'aise avec la présentation d'un support de cours, puis savoir comment l'animer avec un tableau blanc numérique pour faire un remue-méninges, ou savoir diviser le groupe et passer dans des salles virtuelles pour animer le groupe avec des activités en sous-groupes s'apprend 
en pratiquant. Petit à petit, il devient assez naturel d'animer en prenant en compte le fil de discussion qui permet les questions des étudiants sur le thème traité et les réponses des étudiants à nos questions.

- Leçon 2 : adapter l'animation en fonction des objectifs pédagogiques. En parallèle des outils, nous avons travaillé sur l'adaptation de nos méthodes : comment faire ce que l'on faisait dans une salle à travers un écran? Une réunion de travail avec un collègue d'un autre établissement spécialiste de l'enseignement à distance nous a permis de comprendre une chose essentielle qui peut paraître évidente ensuite. Notre premier réflexe a été de chercher comment nous pouvions transformer ce que nous faisions en présentiel pour le faire à travers des écrans, alors que finalement, le plus important était de revenir sur nos objectifs pédagogiques et sur ce que nous voulions faire avec les élèves et de là trouver le moyen de le réaliser à travers des écrans. Ainsi, au lieu de chercher comment transformer le jeu de cartes existant pour réaliser une partie de cours, nous avons réalisé que pour atteindre l'objectif pédagogique fixé, passer par une étude de cas à partir de films conviendrait tout à fait. Il a ainsi fallu reconstruire des outils et nous avons pu le faire en lien avec nos visées pédagogiques. Il nous semble que c'est une tendance assez naturelle de vouloir adapter l'activité pédagogique au numérique au lieu de revenir à comment garder ou adapter les objectifs pédagogiques. Focaliser sur l'adaptation des outils éloigne des objectifs et peut parasiter la réflexion. Repartir des objectifs du cours permet de positionner les instruments comme outils pour réaliser un cours et atteindre un objectif. Les outils numériques sont là pour permettre l'atteinte des objectifs pédagogiques visés et non comme un objectif en soi. Il ne s'agit pas d'utiliser Zoom pour utiliser Zoom mais de prendre cet outil (même s'il est imposé et non choisi) comme un moyen pour atteindre un objectif pédagogique. Si nous n'avions pas classé ces leçons par ordre d'apparition dans la gestion de notre transformation numérique, nous mettrions volontiers celle-ci en tout premier. Il s'agit d'adapter les outils aux objectifs et non pas de s'adapter aux outils.

- Leçon 3 : fédérer une communauté dispersée dans l'espace. Une fois nos cours conçus, nous avons commencé à les animer... À distance, la communauté reste la même mais elle n'est plus en présentiel, donc elle ne peut plus interagir à partir d'éléments physiques, en se regroupant côte à côte. Les apartés sont moins évidents et se font seulement par clavardage ou par un deuxième moyen. C'est moins facile que si l'on est à côté. Les pauses se font chacun chez soi et non plus autour d'un café. La présence physique dans un même lieu permet au corps de s'exprimer. Le non verbal complète les échanges verbaux. La communauté dans ce type d'enseignement devient virtuelle. Ainsi, dans le cas de cours sur Zoom, il est important de constituer une communauté virtuelle avec des personnes dispersées. Cette nécessité de faire et de laisser vivre la communauté se traduit notamment dans les retours des étudiants qui ont majoritairement apprécié les travaux en sous-groupes. Être répartis dans des sous-groupes de façon virtuelle semble avoir permis des échanges entre eux et a donc autorisé des échanges moins formels et plus spontanés non guidés directement par l'intervenant. À chaque passage dans ces sous-groupes, nous avons pu constater des échanges plus spontanés et des étudiants qui allumaient spontanément tous leur caméra, contrairement à ce qui se faisait en groupe complet.

- Leçon 4 : adapter la règle « se montrer ou rester caché ». Ne pas voir les étudiants et se retrouver face à un écran d'initiales ou de prénoms a amené une remise en question. Ainsi, suivre un cours à distance sur Zoom a changé une règle importante des cours. La règle " voir et être vu » en cours ne s'applique pas à un cours sur Zoom car les caméras et les micros des 
étudiants ont été désactivés pour tous avec l'idée de ne pas créer d'interférence au démarrage du cours. Et cela induit que l'enseignant se retrouve vite à démarrer le cours micros et caméras fermés. Mais est-ce la bonne solution? L'enseignant s'affiche et affiche même son intérieur personnel et laisse les étudiants décider. C'est en tout cas le parti que nous avons pris pour minimiser les problèmes techniques de bande passante mais... peut-être à tort. Selon les retours des étudiants, l'affichage est plutôt vu comme positif : " Très bien de demander d'allumer la caméra ça nous implique plus dans le cours. » La distance semble plus importante entre l'enseignant et entre les étudiants si la caméra est éteinte d'un côté. Après réflexion et à partir de cette expérience, nous invitons à privilégier les cours avec « caméra ouverte » et, en cas de problèmes techniques, à demander aux étudiants de mettre une photo avec leur nom sur leur « carré noir ». Nous pensons aussi qu'il est nécessaire de prévoir des temps d'échanges insérés dans le cours au cours desquels la règle est «on ouvre les caméras ».

- Leçon 5 : diviser le temps d'apprentissage de façon très visible. Tout en acceptant cette nouvelle configuration de «face à face», à travers les caméras, nous nous sommes confrontés à une autre façon de gérer le temps du cours. La division du travail ressort à travers la nécessité d'un séquençage plus visible de l'organisation du cours. Même si nous avions 1'habitude de rappeler à chaque activité pédagogique l'objectif de celle-ci et son organisation, il semble que cela soit encore plus important pour un cours à distance pour les apprenants. Un des éléments explicatifs est qu'en présentiel, il est plus facile d'adapter le temps en direct avec les étudiants alors qu'à distance, il nous a semblé plus difficile de revenir sur le temps établi. Par exemple, vous attribuez un temps à une activité, comme le visionnage d'une vidéo. Chacun « repart » avec le temps donné et revient au temps indiqué. Il est donc plus difficile de raccourcir ou de rallonger une activité auprès d'un groupe dispersé à distance. Ainsi, pour gérer ce temps, nous avons choisi d'indiquer sur les diapositives partagées les temps consacrés à chaque activité et nous les rappelions à chaque lancement de l'activité.

- Leçon 6 : favoriser les interactions dans la communauté. En ce qui concerne l'interaction entre apprenants et enseignants, l'implication des étudiants est différente ou différemment perçue. Pour les étudiants, la façon de se montrer, de montrer ce qu'ils savent, et les occasions d'interagir avec les autres et avec l'enseignant doivent être pensées pour permettre aux interactions d'être faciles et fluides. Mobiliser des temps d'échanges avec les étudiants implique encore plus de laisser du temps aux questions afin qu'elles soient exprimées et d'accepter les silences avant les premières questions. Les questions des apprenants peuvent aussi passer par un outil proposé : le fil de discussion. Tout ceci nous a semblé plus difficile qu'en présentiel. Le partage du groupe sur des activités avec la création de questions traitées en sous-groupes dans des salles virtuelles favorise aussi des temps d'échanges ou l'informel s'invite, où les caméras sont allumées de façon naturelle. L'animation de la communauté d'apprenants en présence se fait à travers des outils numériques (salle virtuelle, caméra, fil de discussion, prise de notes en commun...).

\section{Des leviers qui se heurtent à des questions de temps et de lieux}

Ces six leçons tirées de l'expérience sont les points sur lesquels nous avons acquis quelques certitudes et qui pourraient nous servir de base pour continuer une telle expérience. Nous proposons de reprendre ces leçons sur le schéma de l'activité pour en tirer les leviers d'une 
transformation d'un enseignement $100 \%$ en présentiel à un enseignement $100 \%$ à distance (figure 2).

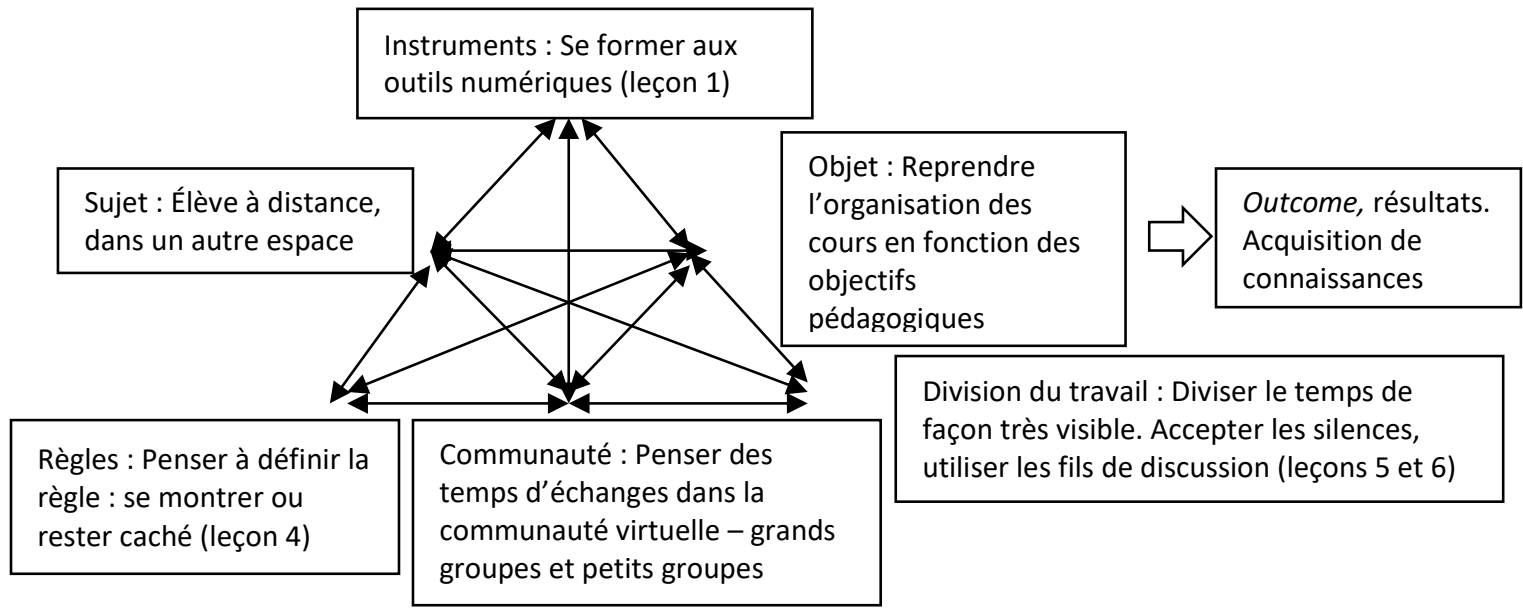

Figure 2

Du présentiel au distanciel : leviers d'une transformation de l'enseignement

Passer d'un enseignement dans une classe physique à un enseignement dans une classe virtuelle exige de revoir tous les éléments de l'activité pour permettre le résultat visé et l'activité « réaliser un cours » pour l'acquisition de connaissances. Nous mettons ainsi en évidence l'importance de penser à tous les éléments articulés de l'activité. Permettre l'acquisition de connaissances dans un cours à distance à travers un outil tel que Zoom implique la nécessité pour l'enseignant de reprendre l'organisation des cours en fonction des objectifs pédagogiques et des possibles offerts par l'outil pour animer un groupe «classe » en repensant la règle « être vu ou entendu » et les possibilités d'échanges au sein de cette communauté à l'aide d'une répartition du temps, partagée avec les apprenants. Cette transformation oblige l'enseignement à se former aux possibilités de l'outil numérique utilisé.

Mais ces leçons sont à compléter par quelques questions qui restent en suspens à la suite de cette expérience. Ce cours étalé sur trois mois n'a pas permis de cerner toutes les transformations réalisées ou à réaliser pour atteindre une pleine satisfaction et une maîtrise de la situation. Ainsi, voici quelques questions encore à traiter.

La division du travail pour l'apprenant est revue dans le cadre d'un enseignement à distance. Les cours numériques induisent-ils des temps différents en fonction de lieux différents? Dans le cadre de notre formation d'ingénieurs, un questionnaire des $1^{\text {res }}$ années (équivalent bac +3 ) réalisé à leur initiative a fait ressortir des difficultés de concentration et d'organisation lors du confinement. L'organisation du temps d'apprentissage et de son organisation sur des lieux hors établissement a induit de réels problèmes dans la poursuite de la formation. Élément exceptionnel dans notre formation d'ingénieurs, la situation de certains étudiants a été qualifiée de décrochage scolaire par les responsables de formation, chargés du suivi des étudiants. Nous pouvons peut-être supposer que ces difficultés sont en partie dues au passage d'un temps en présentiel, qui oblige les étudiants à se lever et à être présents en cours dans un même lieu, à un temps où ils pouvaient faire acte de présence en se connectant tout en étant dans des lieux différents pas forcément destinés à la formation et parfois partagés. La question de l'absentéisme en cours avec ce type de dispositif pédagogique reste en suspens et serait à approfondir. Cette notion de temps appréhendé de manière différente dans cette modalité pédagogique à distance renvoie à la division du travail dans l'activité. Même si nous n'avons pu en tirer une leçon, nous 
estimons important de se poser des questions sur la façon de diviser le temps d'apprentissage et de considérer l'apprenant face à de nouvelles exigences de gestion de son temps de vie. Peut-être pouvons-nous considérer que le lieu « école » se retrouve fractionné dans différents lieux où des apprenants sont en situation d'apprentissage avec l'obligation de structurer leur temps d'enseignement?

La gestion du temps est déjà présentée comme une difficulté pour les étudiants dans les dispositifs à distance (Rouissi et Mottet, 2018). Ainsi, du côté des étudiants, la capacité à gérer son temps de travail est corrélée avec la réussite dans la formation. Da Costa Cabral et al. (2020) invitent à revoir notre conception du déroulement de la formation, de la prise en compte du temps aussi bien du côté formateur que du côté apprenants. Faut-il pour autant prévoir un séquençage différent des cours? Nous avons eu, du côté enseignant, la perception d'un temps qui se déroulait différemment à travers les écrans. C'était comme si le temps prenait plus de temps. Dans l'animation des cours, nous avons dû prendre en compte des temps de régulations techniques par exemple : "Quant au "rythme" en soi, le dispositif "autorise" dans le sens d'Ardoino (2000) les étudiants à fluer, c'est-à-dire à tenir le volant et le boîtier de vitesse de leur(s) propre(s) manière(s), et de changer de postures dans l'action de la situation, dans la "triade Agent-Acteur-Auteur" du même Ardoino » (Da Costa Cabral et al, 2020, p.83). Un lâcher-prise sur le temps devrait donc être réalisé par l'enseignant pour accepter ce qu'autorise ce type de dispositif.

Cette façon de faire cours augmente-t-elle la fatigabilité des enseignants et des étudiants? Lollia et Issaieva (2020) montrent que le manque de temps et l'augmentation de la charge de travail sont soulignés par les enseignants pendant cette période du confinement. Barville et al. (2020) indiquent que ce travail forcé à domicile et à distance a entraîné une hyperconnectivité, une infobésité et une désynchronisation des actions qui fatiguent. Se retrouver seul pour gérer son activité et les nouveautés, pour s'adapter et tenter de bien faire son travail malgré tout a pu entraîner une fatigue psychique. Fosslien et West Duffy (2020) expliquent que les interactions par outils de visioconférence sollicitent plus notre attention et obligent les travailleurs à gérer leur travail et leur environnement personnel. Le fait de pouvoir faire plusieurs choses en même temps réduirait la concentration et augmenterait la fatigue. Nous serions également plus sollicités par les environnements des personnes que l'on verrait à travers les écrans. C'est ainsi que la «Zoom fatigue » se serait imposée à nous et aux étudiants. Les difficultés liées aux problèmes de connexion ressortent également.

Quelles réponses apporter à ces questions? Faut-il fixer de nouvelles règles spécifiques à ce type d'enseignement? Les solutions pourraient se trouver dans la variation de la durée des séances et dans l'utilisation d'une combinaison d'outils. Aller vers plus des séances plus courtes d'une heure à une heure et demie en direct sur Zoom et utiliser les plateformes Moodle pour varier les activités pédagogiques sont peut-être des voies à explorer. Réfléchir à une organisation des cours entre ce qui peut être réalisé en synchrone et ce qui peut l'être en asynchrone en est une autre. Ainsi, les échanges entre l'enseignant et les étudiants ou entre étudiants pourraient être organisés en temps réel par clavardage ou par conférence Web ou visioconférence. L'enseignant animerait ces temps de formation synchrones en maîtrisant l'accès à tel ou tel support et tels documents partagés. Les temps asynchrones se feraient sans temps de connexion simultanée, mais par des accès à des documents sur des plateformes et la mise à disposition de forums et l'utilisation de courriels pour échanger. 


\section{Conclusion}

En premier lieu, le passage au tout numérique analysé dans cet article est bien lié à une situation sanitaire exceptionnelle et au confinement total décidé par l'État français. La décision de basculer vers l'enseignement tout numérique à distance a été voulue pour assurer la continuité pédagogique de notre activité de formation. Nous avons volontairement choisi de tirer des éléments de cette expérience sur ce qui pourrait être retenu pour la suite.

Nous proposons ainsi six leçons tirées de cette expérience. Celles-ci peuvent servir de feuille de route à un établissement pour accompagner ses enseignants dans l'évolution vers un enseignement à distance. Elles apportent également des éléments à évaluer pour un retour d'expérience après le passage à un enseignement à distance pour une équipe pédagogique. Enfin, elles fournissent des éléments de réflexion pour des enseignants sur ce qui a pu fonctionner ou non dans ce type de passage du présentiel à la distance. Former à travers un cours reste bien le but principal d'une activité pédagogique et, à partir de là, il convient que les enseignants se forment aux outils numériques accessibles pour pouvoir définir comment adapter l'animation en fonction des objectifs pédagogiques. Pendant l'animation du cours à distance, l'enseignant est amené à fédérer une communauté dispersée dans l'espace. Ces activités pédagogiques s'attacheront à ramener sur l'écran partagé des éléments discutés pour fédérer en cohérence avec l'objectif pédagogique fixé. L'enseignant a également pour rôle d'adapter et de s'adapter à la règle «en cours, on se montre ou on peut rester caché ». Dans un cours à distance en mode synchrone, le temps d'apprentissage est à diviser de façon très visible et il devient primordial de favoriser les interactions dans la communauté avec des activités pédagogiques en groupe complet et des activités en petits groupes. Approfondir les questions de la perception du temps, de la gestion de la fatigue générée par les outils numériques et de l'organisation de temps synchrones et asynchrones reste nécessaire.

Nous proposons également de poursuivre l'analyse avec des expériences d'autres collègues dans d'autres types d'enseignement, notamment de ceux qui disent "n'avoir rien changé à leur cours » et dans d'autres types d'établissements. Les enseignants habitués à utiliser les outils de collaboration et les technologies spécialisées se sont peut-être trouvés moins dépourvus et plus à l'aise pour basculer vers un enseignement à distance largement numérique. Peut-être retrouverions-nous une adaptation des cours en lien avec les trois types de profils définis par Collin et al. (2018). Il serait aussi intéressant d'avoir une vision plus approfondie des étudiants et de leur perception de cette expérience.

Au mois de juin 2020, nous avons été invités à préparer une rentrée qui se ferait de manière hybride. Il nous semble qu'elle est à construire en alliant les deux modèles, celui plus connu de la formation en présentiel et celui, testé et à confirmer, de la formation à distance réalisée à travers le numérique.

\section{Références}

Ardoino, J. (2000). Les avatars de l'éducation : problématiques et notions en devenir. Presses universitaires de France.

Barville, N., Bétin, P., Cuvillier, B. et de la Gardette, G. (2020). Tous en travail collaboratif... À distance! Entre éthique du collaboratif et brouillage des codes. La revue des conditions de travail, (10). http://anact.fr/... 
Collin, S., Pellerin, G., Blanchard, A., Cordelier, B. et Saffari, H. (2018). Disparités d'adoption des technologies en pédagogie universitaire : un aperçu empirique. Revue internationale des technologies en pédagogie universitaire, 15(1), 9-23. https://doi.org/10.18162/ritpu-2018-v15n1-02

Da Costa Cabral, F., Gremion, C. et Roblez, A. (2020). Évaluation formatrice, rythmes et travail de groupes à distance. Évaluer -Journal international de recherche en éducation et formation, (hors-série $\mathrm{n}^{\mathrm{o}}$ 1), 77-84. http://journal.admee.org/...

Engeström, Y. (1987). Learning by expanding: An activity-theorical approach to developmental research. Orienta-Konsultit. http://lchc.ucsd.edu/...

Engeström, Y. (2006). L'interagentivité orientée-objet : vers une compréhension de l'intentionnalité collective dans les activités distribuées. Dans J. M. Barbier et M. Durand (dir.), Sujets, activités, environnements (p. 135-174). Presses Universitaires de France.

Fosslien, L. et West Duffy, M. (2020, 29 avril). How to combat Zoom fatigue. Harvard Business Review. http://hbr.org/...

Fourgous, J.-M. (2010). Réussir l'école numérique. Rapport de la mission parlementaire de Jean-Michel Fourgous, député des Yvelines, sur la modernisation de l'école par le numérique. Vie publique. http://vie-publique.fr/...

Karsenti, T. (2018). Portrait d'enjeux actuels du numérique en pédagogie universitaire : entre les MOOCs et le e-sport. Revue internationale des technologies en pédagogie universitaire, 15(1), 5-8. https://doi.org/10.18162/ritpu-2018-v15n1-01

Lebrun, M. (2007). Théories et méthodes pédagogiques pour enseigner et apprendre. Quelle place pour les TIC dans l'éducation? ( $2^{\mathrm{e}}$ éd.). De Boeck Université.

Lollia, M. et Issaieva, E. (2020). Comment les enseignants assurent la continuité pédagogique et évaluent en contexte de pandémie? Une étude en Guadeloupe. Évaluer - Journal international de recherche en éducation et formation, (hors-série $n^{0} 1$ ), 181-192. http://journal.admee.org/...

Mines Saint-Étienne (s.d.). Ingénieur civil des mines. Récupéré le 18 juin 2020 de http://mines-stetienne.fr/...

Ministère de l'Enseignement supérieur, de la Recherche et de l'Innovation. (2020, 17 avril). Classes préparatoires aux grandes écoles (C.P.G.E.). Internet Archive. https://web.archive.org/...

Rouissi, S. et Mottet, M. (2018). Gestion du temps, profils et réflexions d'étudiants inscrits dans un cours en ligne à leur premier trimestre universitaire. Revue internationale des technologies en pédagogie universitaire, 15(1), 24-33. https://doi.org/10.18162/ritpu-2018-v15n1-03

Vygotski, L. (2002). Pensée et langage (F. Sève, trad.) La dispute. (Ouvrage original publié en 1934.) 